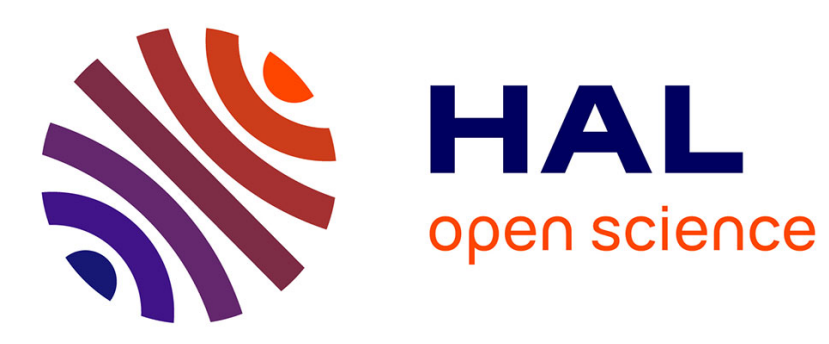

\title{
Locally Parallel Textures Modeling with Adapted Hilbert Spaces
}

Pierre Maurel, Jean-François Aujol, Gabriel Peyré

\section{To cite this version:}

Pierre Maurel, Jean-François Aujol, Gabriel Peyré. Locally Parallel Textures Modeling with Adapted Hilbert Spaces. Energy Minimization Methods in Computer Vision and Pattern Recognition 2009, Aug 2009, Bonn, Germany. pp.429-442, 10.1007/978-3-642-03641-5 . hal-00392270

\section{HAL Id: hal-00392270 \\ https://hal.science/hal-00392270}

Submitted on 6 Jun 2009

HAL is a multi-disciplinary open access archive for the deposit and dissemination of scientific research documents, whether they are published or not. The documents may come from teaching and research institutions in France or abroad, or from public or private research centers.
L'archive ouverte pluridisciplinaire HAL, est destinée au dépôt et à la diffusion de documents scientifiques de niveau recherche, publiés ou non, émanant des établissements d'enseignement et de recherche français ou étrangers, des laboratoires publics ou privés. 


\title{
Locally Parallel Textures Modeling with Adapted Hilbert Spaces
}

\author{
Pierre Maurel ${ }^{1}$, Jean-François Aujol ${ }^{1}$, and Gabriel Peyré ${ }^{2}$ \\ 1 CMLA, ENS Cachan, CNRS, UniverSud, 61 avenue du Président Wilson, 94235 \\ Cachan Cedex, France \\ 2 Ceremade, Université Paris-Dauphine, Place du Maréchal De Lattre De Tassigny, \\ 75775 Paris Cedex 16, France
}

\begin{abstract}
This article ${ }^{3}$ presents a new adaptive texture model. Locally parallel oscillating patterns are modeled with a weighted Hilbert space defined over local Fourier coefficients. The weights on the local Fourier atoms are optimized to match the local orientation and frequency of the texture. We propose an adaptive method to decompose an image into a cartoon layer and a locally parallel texture layer using this model and a total variation cartoon model. This decomposition method is then used to denoise an image containing oscillating patterns. Finally we show how to take advantage of such a separation framework to simultaneously inpaint the structure and texture components of an image with missing parts. Numerical results show that our method improves state of the art algorithms for directional and complex textures.
\end{abstract}

\section{Introduction}

The analysis and modeling of textures is a central topic in computer vision and graphics. Texture modeling is fundamental for a large number of problems, such as image segmentation, object recognition and image restoration.

\subsection{Previous Works}

Image Decomposition. A variational decomposition algorithm seeks a decomposition $f=u+v$ of an image $f$ where $u$ should capture the sketch of the image and $v$ the texture content. This decomposition is often defined as the solution of a minimization problem involving two norms, one for each component. Total variation [1] is broadly used as a cartoon model since it allows to recover piecewise smooth functions without smoothing sharp discontinuities. On the other hand, the norm on $v$, the texture component, should be small for typical texture patterns one wants to extract.

Following [1], where Rudin, Osher and Fatemi proposed to capture the noise of an image by using the usual $\mathrm{L}^{2}$ norm, Yves Meyer [2] pushed forward the idea

\footnotetext{
3 This work has been done with the support of the French "Agence Nationale de la Recherche" (ANR), under grant NATIMAGES (ANR-08-EMER-009), "Adaptivity for natural images and texture representations".
} 
of using more complex norms $\|\cdot\|_{\mathrm{T}}$ to capture oscillating patterns. In particular he proposed a weak norm dual of the TV norm. This idea inspired several works [3-5]. An alternative to this dual norm approach has been presented in [6], the Morphological Component Analysis: it uses the $\ell^{1}$ norm of decompositions on bases such as a local cosine dictionary for the texture component and a wavelet dictionary for the cartoon one.

Inpainting. The problem of inpainting can be stated as follows : given a region $\Omega$ to be restored, use the valid surrounding information for synthesizing the most plausible data in $\Omega$. Several classes of methods have been considered. In the first category of approaches, the focus has been on recovering the geometry. These methods [7-11] use partial differential equations that propagate the information from the boundary of the missing region to its interior. The drawback of this kind of methods is their well-known incapacity to restore texture. In parallel to these geometry-oriented approaches, the exemplar-based methods $[12,13]$ turned out to be very efficient for reconstructing isotropic and non-geometric textures. Different approaches have been proposed in combination with an exemplar-based inpainting, either based on a manual intervention by the user [14], or trying to combine texture and geometric interpolation in the most automated possible way [15]. A last class of approaches relies on sparse regularization in several transform domains (e.g. Fourier, wavelet or framelet) and also aims to deal with geometric and texture information simultaneously $[16,17]$.

\subsection{Contributions}

The main contribution of this work is a new adaptive texture model. We propose methods for using this new model in some applications such as image decomposition, denoising and inpainting, and we present algorithms for solving these problems. We model locally parallel textures in order to extract oscillating patterns which present spatial and frequency variability. We start (section 2) by defining a texture norm $\|\cdot\|_{\mathrm{T}}=\|\cdot\|_{\xi}$ depending on a parameter $\xi(x)$ which is the instantaneous frequency of the oscillating texture. For a point $x$ in the image, $\xi(x)$ gives the local frequency $\|\xi(x)\|$ and the local orientation $\xi(x) /\|\xi(x)\|$ of the texture around $x$. The norm $\|\cdot\|_{\xi}$ is small for an oscillating pattern around $x$ if its main frequency is close to $\xi(x)$. We then use this norm for a decomposition problem (section 3): we want to separate the image into three layers, $f=u+v+w$ where $u$ is the geometric layer, $v$ is the texture modeled by our norm and $w$ is the noise. And finally the interest of such a texture norm is highlighted in section 4 by its use in an inpainting method which simultaneously inpaints the geometric and the texture layers. Numerical examples are shown for decomposition, denoising and inpainting and our results are compared with other methods.

In the following, we suppose that $f \in \mathbb{R}^{N}$ is a discrete image of $N=n \times n$ pixels and the two operators gradient and divergence are discretized by forward finite difference (for example, we refer the reader to [18] for details). In this framework, we have $\|\nabla\|=\sqrt{8}$. 


\section{Texture Modeling Using an Adaptive Hilbert Norm}

\subsection{Hilbert Texture Norm}

In [18], Aujol and Gilboa proposed to use a linear Hilbert norm defined by some symmetric positive kernel $K:\|v\|_{\mathrm{T}}^{2}=\langle K v, v\rangle_{\mathrm{L}^{2}}$. This norm can be computed using a frame $\left\{\psi_{\ell}\right\}_{\ell}$ that is a possibly redundant family of $P \geqslant N$ atoms $\psi_{\ell} \in$ $\mathbb{R}^{N}$. The decomposition of an image in this frame reads

$$
\Psi f=\left\{\left\langle f, \psi_{\ell}\right\rangle\right\}_{\ell=0}^{P-1} \in \mathbb{R}^{P},
$$

where $\Psi: \mathbb{R}^{N} \rightarrow \mathbb{R}^{P}$ is the frame operator.

Given a set of positive weights $\gamma_{\ell} \geqslant 0$, a norm can then be defined as

$$
\|f\|_{\mathrm{T}}^{2}=\sum_{\ell} \gamma_{\ell}^{2}\left|\left\langle f, \psi_{\ell}\right\rangle\right|^{2}=\|\gamma \Psi f\|_{L^{2}}^{2}
$$

where $\gamma=\operatorname{diag}_{\ell}\left(\gamma_{\ell}\right)$. This corresponds to a Hilbert space associated to the kernel $K=\Psi^{*} \gamma^{2} \Psi$.

\subsection{Texture Norm Over a Local Fourier Basis}

The original construction in [18] uses the Fourier basis so that $\Psi$ corresponds to the discrete Fourier transform. This defines a translation-invariant kernel $K$. This paper proposes to replace the global Fourier basis by a redundant local Fourier basis, to capture the spatially and frequentially varying structures of locally parallel textures.

Local Fourier Frame A discrete short time Fourier atom, located around a position $x_{p}=p \Delta_{x}$ and with local frequency $\xi_{k}=k \Delta_{\xi}=k / q$ is defined as

$$
\psi_{p, k}[y]=q^{-1} g\left[y-p \Delta_{x}\right] e^{\frac{2 i \pi}{q}\left(y_{1} k_{1}+y_{2} k_{2}\right)},
$$

for $k \in\{-q / 2, \ldots, q / 2-1\}^{2}$ and $p \in\left\{0, \ldots, n / \Delta_{x}\right\}^{2}$, where $g$ is a smooth window, centered around 0 , and the size of its support is $q \times q$ pixels with $q>\Delta_{x}$. In this paper, we use a Haning window function: $g[x]=\sin \left(\pi x_{1} / q-\right.$ $\pi / 2)^{2} \sin \left(\pi x_{2} / q-\pi / 2\right)^{2}$.

The local Fourier frame $\left\{\psi_{p, k}\right\}_{p, k}$ is a redundant family of $P=\left(q / \Delta_{x}\right)^{2} N$ vectors of $\mathbb{R}^{N}$. The decomposition $\Psi f=\left\{\left\langle f, \psi_{p, k}\right\rangle\right\}_{p, k} \in \mathbb{R}^{P}$ of an image $f$ in this frame can be computed with the 2D Fast Fourier Transform of the $q \times q$ image $f[y] g\left[\Delta_{x} p-y\right]$. The computation of $\Psi f$ thus requires $O\left(N Q \log _{2}(Q) / \Delta_{x}^{2}\right)$ operations.

The dual operator $\Psi^{*}$ reconstructs an image $\Psi^{*} c \in \mathbb{R}^{N}$ from a set of coefficients $c[p, k] \in \mathbb{R}^{Q \times N}$

$$
\Psi^{*} c=\sum_{p, k} c[p, k] \psi_{p, k}
$$


This dual operator is implemented using $N / \Delta_{x}^{2}$ inverse Fast Fourier Transforms. The operator $\Psi^{*} \Psi$ is in fact diagonal, and one has

$$
\Psi^{*} \Psi=\operatorname{diag}_{x}\left(\sum_{y} g^{o}\left[\Delta_{x} y-x\right]^{2}\right) .
$$

and the norm of the operator $\Psi^{*} \Psi$ is $\max _{x} \sum_{y} g^{o}\left[\Delta_{x} y-x\right]^{2}$.

\subsection{Weight Design}

We define a Hilbert norm $\|\cdot\|_{\mathrm{T}}$ adapted to oscillating texture as a weighted norm over the local Fourier coefficients. The general formulation (2) is instantiated using a local Fourier frame $\psi_{\ell}=\psi_{p, k}$ for $\ell=(p, k)$ as follow

$$
\|f\|_{\mathrm{T}}^{2}=\sum_{p, k} \gamma_{p, k}^{2}\left|\left\langle f, \psi_{p, k}\right\rangle\right|^{2},
$$

where each $\gamma_{p, k} \geqslant 0$ weights the influence of each local Fourier atom in the texture model.

Intuitively, $\gamma_{p, k}$ should be small when the texture $f$ contains a local oscillation of frequency close to $\xi_{k}$ around the point $x_{p}$. We consider a locally oscillating texture model, where typical texture patterns are locally well approximated by a single atom.

The texture norm $\|\cdot\|_{\mathrm{T}}$ is therefore parametrized by a vector field $\xi: \mathbb{R}^{N} \mapsto \mathbb{R}^{2}$ which represents the local frequency of the texture component of $f$. For a point $x$ of the image, the local frequency around $x$ is given by $|\xi(x)|$ and the local orientation of the texture is given by $\xi(x) /|\xi(x)|$. The norm $\|\cdot\|_{\mathrm{T}}=\|\cdot\|_{\xi}$ should be small for an oscillating pattern around the point $x$ if its main frequency is close to $\xi(x)$. As a consequence the weight $\gamma_{p, k}$ should be small if $\xi_{k}$ is close to $\xi\left(x_{p}\right)$ or to $-\xi\left(x_{p}\right)$. By convention, $\xi(x)$ is set to $(0,0)$ if there is no significant oriented patterns around $x$ in the image.

The weights are therefore defined as a function of $\xi$ :

$$
\gamma_{p, k}(\xi)=\left\{\begin{array}{c}
1 \text { if } \xi\left(x_{p}\right)=(0,0) \\
\left(1-G_{\sigma}\left(\left\|\xi_{k}+\xi\left(x_{p}\right)\right\|\right)\right)\left(1-G_{\sigma}\left(\left\|\xi_{k}-\xi\left(x_{p}\right)\right\|\right)\right) \text { otherwise }
\end{array}\right.
$$

where $\left.G_{\sigma}(x)=\exp \left(-(x / \sigma)^{2} / 2\right)\right)$ and $\sigma$ is a scale parameter reflecting the deviation we are expecting to find in the frequency spectrum of the texture compared to $\xi(x)$ (in our numerical experiments we took $\sigma=1$ ). When there is not a significant oriented texture around $x_{p}$, we choose $\gamma_{p, k}=1$ for all $k$, in order not to promote an arbitrary orientation in the extraction. The texture norm is finally given by:

$$
\|v\|_{\mathrm{T}}^{2}=\|v\|_{\xi}^{2}=\sum_{p, k} \gamma_{p, k}(\xi)^{2}\left|\left\langle v, \psi_{p, k}\right\rangle\right|^{2}=\|\Gamma(\xi) \Psi v\|_{L^{2}}^{2} .
$$

where $\Gamma(\xi)=\operatorname{diag}_{\ell=(p, k)}\left(\gamma_{p, k}(\xi)\right)$. This is actually a semi-norm since $\|v\|_{\mathrm{T}}=0$ does not imply $v=0$ but, for the sake of simplicity, we use the term of norm in the following. 


\section{Image Decomposition and Denoising Using an Adaptive Hilbert Norm}

Decomposing an image into meaningful components is an important problem in image processing. Using the texture norm introduced in section 2 , we present an image decomposition framework which aims to separate an image $f$ into three components: $f=u+v+w$, where $u$ should capture the sketch of the image, $v$ the texture content and $w$ the noise. We define this decomposition as the solution of the following minimization problem:

$$
(u, v, \xi)=\underset{\tilde{u}, \tilde{v}, \tilde{\xi} \in \mathcal{C}}{\operatorname{argmin}} \mu\|\tilde{v}\|_{\tilde{\xi}}^{2}+\lambda\|\tilde{u}\|_{\mathrm{TV}}+\frac{1}{2}\|f-\tilde{u}-\tilde{v}\|_{L^{2}}^{2}, \quad w=f-u-v .
$$

where \|\|$_{\mathrm{TV}}$ is the total variation norm, $\|u\|_{\mathrm{TV}}=\int|\nabla u|$ (the discrete total variation of $u$ is then defined by $\left.\|u\|_{\mathrm{TV}}=\sum_{1 \leqslant i, j \leqslant n}\left|(\nabla u)_{i, j}\right|\right)$ and $\|v\|_{\xi}$ is our texture norm defined by (8).

$\mathcal{C}$ is a set of constraints on the orientation field $\xi$. We first force the frequency $|\xi|$ to be large enough in order not to extract low frequencies in the texture component $v: \forall p,\left|\xi\left(x_{p}\right)\right|>\tau$, for some real positive parameter $\tau>0$. Furthermore, an oscillating pattern of frequency $\xi\left(x_{p}\right)$ is assumed to be present in the image $f$ around the point $x_{p}$ only if $\left|\left\langle f, \psi_{p, k}\right\rangle\right|>\eta_{p}$ where $k=\xi\left(x_{p}\right) / \Delta_{\xi}$ and $\eta_{p}>0$ is a real positive parameter. In fact, one does not want to arbitrary select a frequency for an area of the image where there is no oscillating pattern. In our numerical experiments we take $\tau=2 / q$, where $q$ is the size of the local Fourier windows, and $\eta_{p}=2 \overline{\left|\Psi f_{p}\right|}$ where $\overline{\left|\Psi f_{p}\right|}$ is the average value of $\left|\left\langle f, \psi_{p, k^{\prime}}\right\rangle\right|$ for $k^{\prime} \in\{-q / 2, \ldots, q / 2-1\}^{2}$. In short, we have:

$$
\mathcal{C}=\left\{\begin{array}{l|l}
\xi: \mathbb{R}^{N / \Delta_{x}} \mapsto \mathbb{R}^{2} & \begin{array}{l}
\forall p, \\
\forall p,\left(\forall k,\left|\left\langle f, \psi_{p, k}\right\rangle\right| \leqslant \eta_{p}\right) \Rightarrow \xi\left(x_{p}\right)=(0,0)
\end{array}
\end{array}\right\}
$$

The minimization (9) iterates between two steps: one on $\xi$ and one on $u$ and $v$. We detail these two steps in the next two sections. Although the energy is decreasing at each step, this algorithm is not guaranteed in general to converge to a minimum. However we did not encounter any optimization problems during our numerical experiments.

\subsection{Minimization with respect to the Orientation Field $\xi$}

If $u$ and $v$ are fixed, we search for the frequency field $\xi$ verifying:

$$
\xi=\underset{\tilde{\xi} \in \mathcal{C}}{\operatorname{argmin}}\|v\|_{\tilde{\xi}}^{2}
$$

This requires, for each $p$, to compute:

$$
\xi\left(x_{p}\right)=\underset{\tilde{\xi}\left(x_{p}\right) \in \mathcal{C}}{\operatorname{argmin}} \sum_{k} \gamma_{p, k}\left(\tilde{\xi}\left(x_{p}\right)\right)^{2}\left|\left\langle v, \psi_{p, k}\right\rangle\right|^{2} .
$$


where $\gamma_{p, k}\left(\tilde{\xi}\left(x_{p}\right)\right)$ is given by $(7)$.

If $\sigma$ in the weight definition (7) is small enough, this minimization boils down to compute $\max _{k}\left|\left\langle v, \psi_{p, k}\right\rangle\right|$, which allows us to speed up the computation by taking:

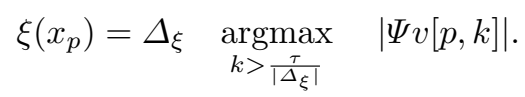

Figure 1 illustrates the underlying principle of this orientation estimation: for a given point $x_{p}$, a unique direction and frequency $\xi\left(x_{p}\right)$ is selected and the corresponding weights $\gamma_{p, k}(\xi)$ are constructed according to (7).

(a)

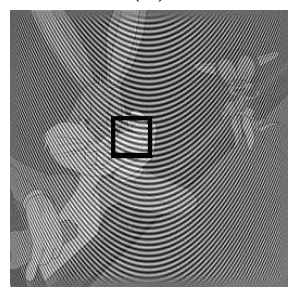

(b)

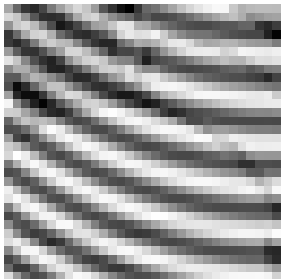

(c)

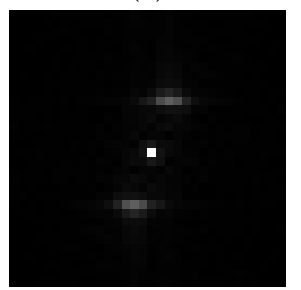

(d)

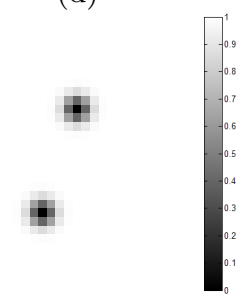

Fig. 1. Illustration of the orientation estimations. From left to right: (a) the input image $f$, (b) the windowed image around some point $x_{p}$, (c) the corresponding local Fourier transform and $(\mathrm{d})$ the weights $\gamma_{p, k}(\xi)$ corresponding to the $\xi$ estimated from the local Fourier transform.

\subsection{Minimization with respect to the Components $u$ and $v$}

If $\xi$ is fixed, we search for $u$ and $v$ verifying:

$$
(u, v)=\underset{\tilde{u}, \tilde{v}}{\operatorname{argmin}} \mu\|\Gamma(\xi) \Psi \tilde{v}\|_{L^{2}}^{2}+\lambda\|\tilde{u}\|_{\mathrm{TV}}+\frac{1}{2}\|f-\tilde{u}-\tilde{v}\|_{L^{2}}^{2},
$$

where $\Gamma(\xi)$ is defined at the end of section 2.3. This minimization is done itself iteratively on $u$ and $v$. Starting from some initial $u^{(0)}$ and $v^{(0)}$, one solves:

- $v$ is fixed: one minimizes

$$
u^{(i+1)}=\underset{\tilde{u}}{\operatorname{argmin}} \lambda\|\tilde{u}\|_{\mathrm{TV}}+\frac{1}{2}\left\|\left(f-v^{(i)}\right)-\tilde{u}\right\|_{L^{2}}^{2} .
$$

This minimization can be solved using iterations of the original algorithm of Chambolle [19]. This algorithm is based on the observation that $u^{(i+1)}=$ $\left(f-v^{(i)}\right)+\lambda \operatorname{div}(w)$ where $w$ is the solution of the following constrained minimization problem

$$
w=\underset{\|\tilde{w}\|_{\infty} \leqslant 1}{\operatorname{argmin}}\left\|\left(f-v^{(i)}\right)+\lambda \operatorname{div}(\tilde{w})\right\|,
$$


where the infinite norm of a vector field $w=\left(w_{1}, w_{2}\right)$ is

$$
\|w\|_{\infty}=\max _{i, j} \sqrt{w_{1}[i, j]^{2}+w_{2}[i, j]^{2}} .
$$

Chambolle proposed a fixed point algorithm to solve (14), and one can also use a projected gradient descent by initializing $w^{(0)}=0$ and then iterating a gradient step

$$
\bar{w}^{(\ell)}=w^{(\ell)}+\nu \nabla\left(\bar{u}^{(k)}+\lambda \operatorname{div}\left(w^{(\ell)}\right)\right)
$$

and a projection on the constraints

$$
\forall(i, j), \quad w^{(\ell+1)}[i, j]=\frac{\bar{w}^{(\ell)}[i, j]}{\max \left(\left\|\bar{w}^{(\ell)}[i, j]\right\|, 1\right)} .
$$

The gradient step size should satisfy $\nu<2 /\|\nabla\|^{2}=1 / 4$ (with the discretization used in this paper) so that $\left.f-v^{(i)}\right)+\lambda \operatorname{div}\left(w^{(\ell)}\right)$ converges with $\ell \rightarrow+\infty$ to $u^{(i+1)}$.

- $u$ is fixed: one minimizes

$$
v^{(i+1)}=\underset{\tilde{v}}{\operatorname{argmin}} \mu\|\Gamma(\xi) \Psi \tilde{v}\|_{L^{2}}^{2}+\frac{1}{2}\left\|\left(f-u^{(i+1)}\right)-\tilde{v}\right\|_{L^{2}}^{2},
$$

Computing the gradient of (18), we obtain that $v^{(i+1)}$ satisfies:

$$
\left(2 \mu \Psi^{*} \Gamma^{2} \Psi+\mathrm{Id}\right) v^{(i+1)}=f-u^{(i+1)}
$$

and the solution can be obtained by conjugate gradient descent (notice that $A=\mu \Psi^{*} \Gamma^{2} \Psi+\operatorname{Id}$ is positive symmetric).

\subsection{Decomposition of a Noise Free Image}

If the input image $f$ does not contain any noise, one can also decompose $f$ into only two components, the sketch $u$ and the texture $f-u$ :

$$
(u, \xi)=\underset{\tilde{u}, \tilde{\xi} \in \mathcal{C}}{\operatorname{argmin}} \lambda\|\tilde{u}\|_{\mathrm{TV}}+\frac{1}{2}\|f-\tilde{u}\|_{\tilde{\xi}}^{2},
$$

In this case, a faster algorithm can be used. The minimization step on $\xi$ is the same as the one described in section 3.1, but the second step on $v$ is different. One can use an extension of Chambolle's algorithm designed to deal with inverse problem, see for instance [20,21] for equivalent description of this method. From an initial texture layer $u^{(0)} \in \mathbb{R}^{N}$, this algorithm iterates between a gradient step of the functional $u \mapsto\|\gamma \Psi u-y\|^{2}$ (where $y=\gamma \Psi f$ ):

$$
\bar{u}^{(k)}=u^{(k)}+\nu \Psi^{*} \gamma\left(y-\gamma \Psi u^{(k)}\right)
$$


where $\nu>0$ is a step size that should obey $\nu<2 /\|\gamma \Psi\|^{2}$, and a denoising step

$$
u^{(k+1)}=\underset{\tilde{u} \in \mathbb{R}^{N}}{\operatorname{argmin}} \frac{1}{2}\left\|\bar{u}^{(k)}-\tilde{u}\right\|^{2}+\lambda \nu\|\tilde{u}\|_{\mathrm{TV}} .
$$

which is equivalent to (13) and therefore can be solved using the projected gradient descent described in section 3.2 .

\subsection{Numerical Examples}

The local Fourier transform described in section 2.2 depends on two parameters $q$, which is the size of the local Fourier windows, and $\Delta_{x}$ which measure the overlapping of the windows. Let us note that an estimation of the lowest frequency $\xi_{\min }$ present in the texture component to extract is an indication for the choice of the parameter $q$. As a matter of fact, if $q$ is too small, the spectrum of the local Fourier windows cannot differentiate very low frequency oscillating patterns from geometric information. In fact, we have $\xi_{k}=k / q$ and we can take $q=3 / \xi_{\min }$ to be sure that $\xi_{\min }$ is detected. As for the parameter $\Delta_{x}$, which verifies $\Delta_{x}<q$, it should be taken smaller for a texture which strongly varies spatially than for a texture which is smoother. Good candidates for $\Delta_{x}$ are for example $q / 2$ or $q / 4$.

Figure 2 presents an example of the decomposition of a noise free image: the input image $256 \times 256$, shown in the first row, is generated by addition of a cartoon picture and a synthetic texture whose orientation and frequency vary spatially. These two components are shown in the first column. We applied the $T V-L^{2}$ method [1] and we chose the smallest parameter $\lambda$ (on the total variation norm) which provides a total extraction of the texture (here $\lambda=0.9$ ). For our method we chose $\lambda=0.1, q=16, \Delta_{x}=4$.

In Figure 3 an image $f$ composed by a cartoon picture and a fingerprint texture is degraded by a Gaussian noise. The noisy image $f$ is then decomposed into three components $u, v$, and $w$ using our method with the following parameters $\lambda=0.1, \mu=0.3, q=16, \Delta_{x}=4$. Since $u$ captures the sketch of the image, $v$ the locally parallel patterns and $w$ the noise, we can reconstruct a restored version of the noisy image by addition of $u$ and $v$.

With the same idea, we show in Figure 4 an example of result obtained by this decomposition and denoising process on the "barbara" image. Figure 5 compares our result with two other denoising methods. Every parameter is chosen to achieve the best SNR result. The decomposition between structure and texture provides a better reconstruction of the texture and therefore a better SNR. Let us remark that the former image decomposition frameworks (such as TV-G [2] or TV $\left.-H^{-1}[4]\right)$ are not suitable for denoising. As a matter of fact, the $G$ and the $H^{-1}$ norms are low for any high-frequency patterns and are then also low for a large part of the noise. On the other hand, the TV norm penalizes strongly oscillating patterns and therefore these models are not able to separate efficiently the texture from the noise. On the contrary our norm is low for patterns which presents a certain frequency and orientation and is therefore more appropriate for denoising. 
(a)

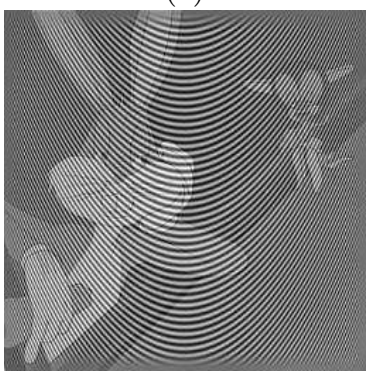

(c)

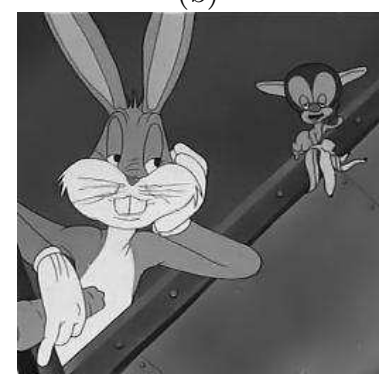

(e)

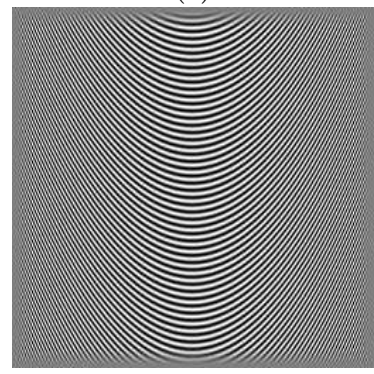

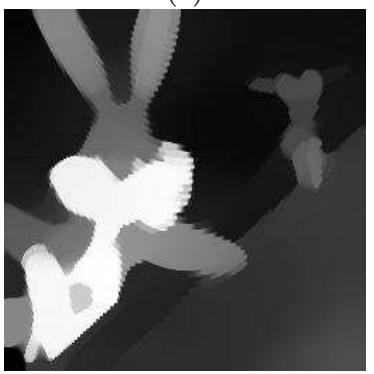

(f)

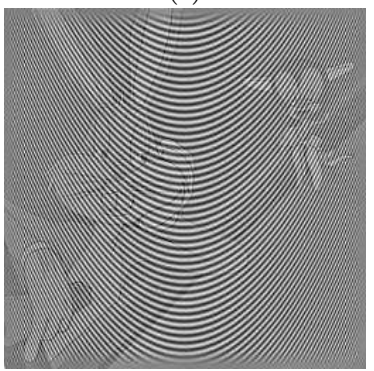

(d)

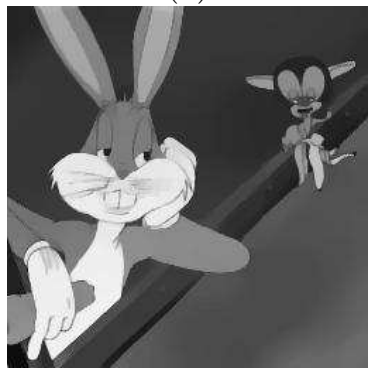

(g)

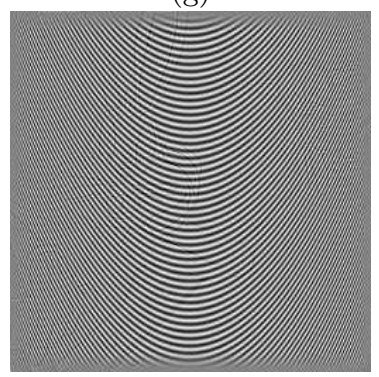

Fig. 2. A synthetic example: (a) the input image, first column: (b) original structure and (e) texture components used to produce the image, second column (c) and (f): decomposition results with $T V-L^{2}$ and third column (d) and (g) : decomposition results with our adapted TV-Hilbert method. The obtained result is almost perfect.

\section{Inpainting With Adapted Hilbert Space}

\subsection{Simultaneous cartoon and texture inpainting}

Inpainting aims at restoring an image $f$ from which a set $\Omega \subset\{0, \ldots, n-1\}^{2}$ of pixels is missing. It corresponds to the inversion of the ill posed linear problem $y=\Phi f+\varepsilon$ where $\Phi$ is defined as

$$
(\Phi f)(x)= \begin{cases}0 \quad \text { if } & x \in \Omega \\ f(x) & \text { if } \quad x \notin \Omega .\end{cases}
$$




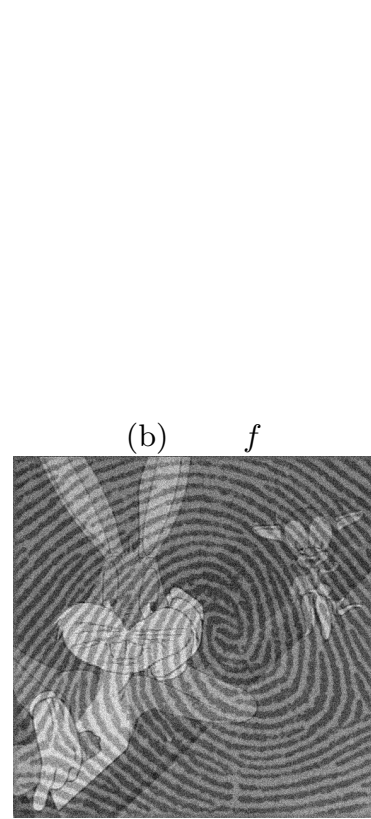

(e)

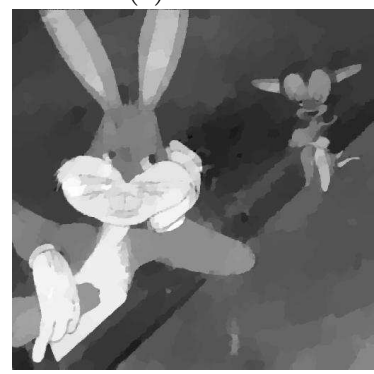

(a) $f_{0}$

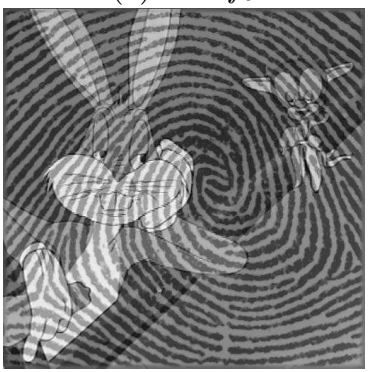

(c) $u+v$

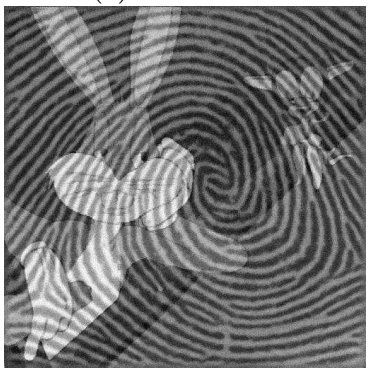

(f) $v$

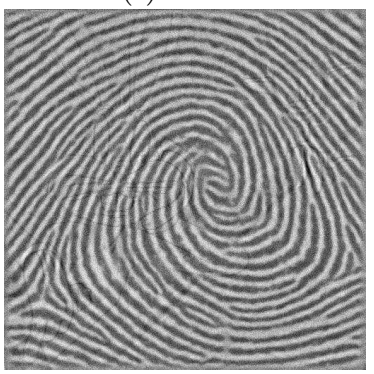

(d) $\quad \xi$

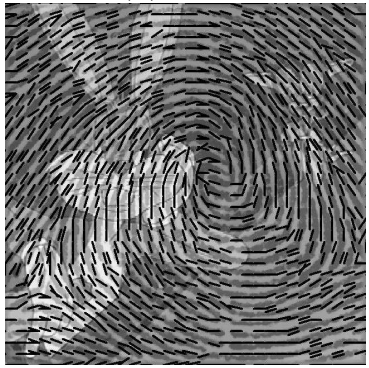

(g)

$w$

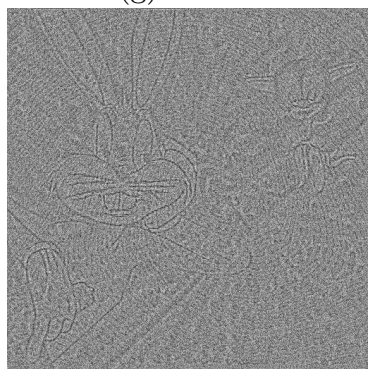

Fig. 3. First row: (a) the original noise free image. Second row: (b) the input noisy image $f$, (c) the restored image $u+v$ and (d) the estimated orientations of oscillating patterns $\xi$. Third row: the decomposition into three components, (e) the sketch $u$ of the image, (f) the texture content $v$ and $(\mathrm{g})$ the noise $w$.

and $\varepsilon$ is an additive noise. We search for the image $f$ as a decomposition $f \approx u+v$ where $u$ has a low total variation and $v$ has a small Hilbert texture norm. This corresponds to the solution of

$$
(u, v, \xi)=\underset{\tilde{u}, \tilde{v}, \tilde{\xi} \in \mathcal{C}}{\operatorname{argmin}} \lambda\|\tilde{u}\|_{\mathrm{TV}}+\mu\|\tilde{v}\|_{\tilde{\xi}}^{2}+\frac{1}{2}\|\Phi(\tilde{u}+\tilde{v})-y\|_{L^{2}}^{2},
$$

where $\mu$ and $\lambda$ should be adapted to the noise level and the regularity of $f$.

The inpainting is done similarly to section 3 by performing the minimization iteratively on $\xi, u$ and $v$. The minimization step on $\xi$ does not change, see 
(a)

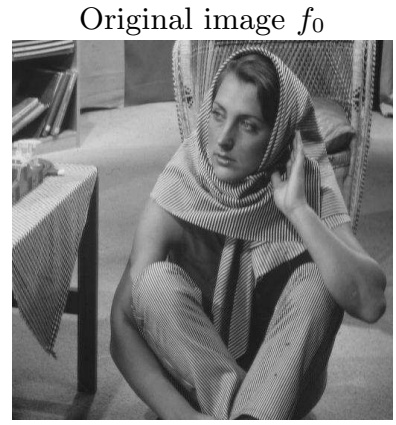

(d) $u$

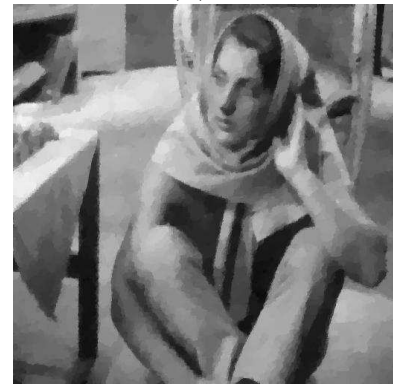

(b) Noisy input image $f$ $(\mathrm{SNR}=10.25)$

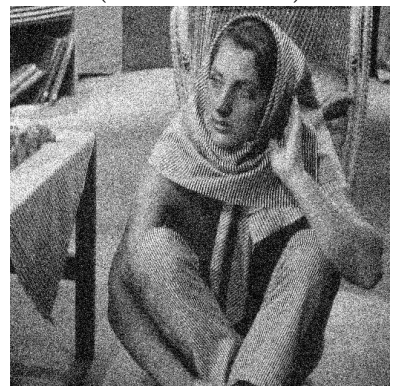

(e) $v$

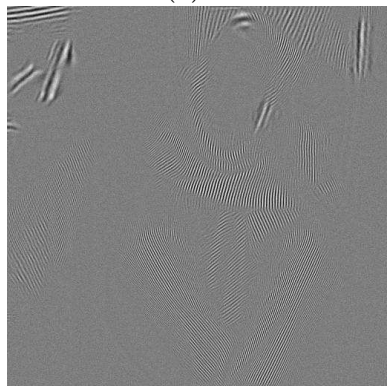

(c)

Restored image $u+v$

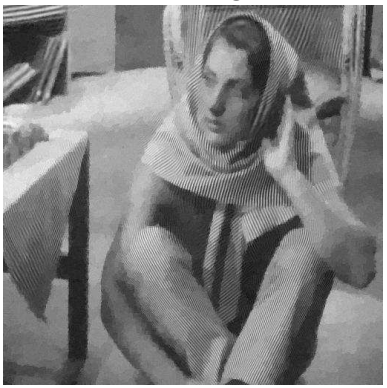

(f) $w$

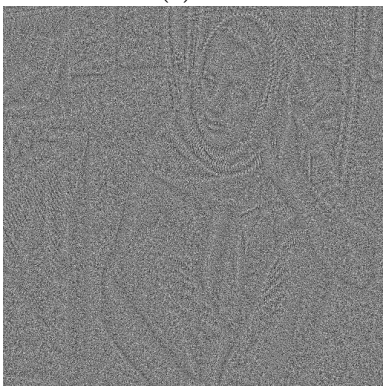

Fig. 4. first row: (a) the original noise free image $f_{0}$, (b) the noisy input image $f$ and (c) the restored image $u+v$. second row: the decomposition (d) $u$ the sketch of the image, (e) $v$ the texture content and (f) $w$ the noise.

(a) $\mathrm{SNR}=17.34$

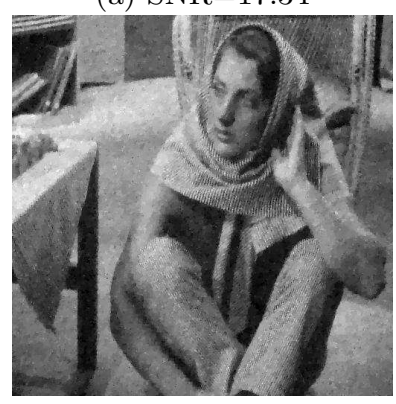

(b) $\mathrm{SNR}=17.98$

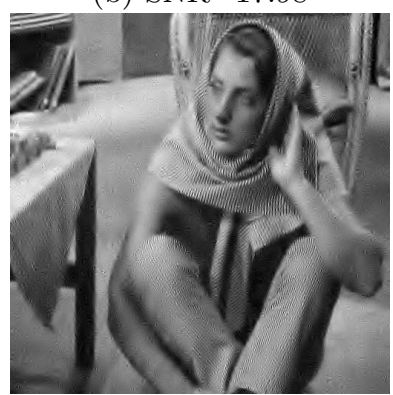

(c) $\mathrm{SNR}=19.93$

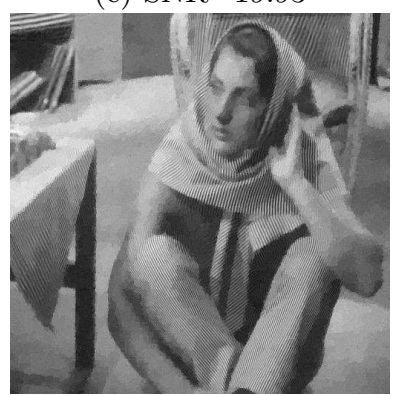

Fig. 5. Comparison with other methods. Denoising of image $f$ from Fig. 4. (a) TVdenoising ( $\lambda$ is chosen to achieve the best SNR, $\lambda=0.1$ ),(b) Translation Invariant Wavelet Denoising (the threshold is chosen to achieve the best SNR) and (c) our result which achieves a better SNR.

section 3.1. We describe here the second step, on $u$ and $v$. Starting from some initial $u^{(0)}$ and $v^{(0)}$, one solves 
- $v$ is fixed: one minimizes

$$
u^{(i+1)}=\underset{\tilde{u}}{\operatorname{argmin}} \lambda\|\tilde{u}\|_{\mathrm{TV}}+\frac{1}{2}\|\Phi \tilde{u}-\bar{y}\|_{L^{2}}^{2}
$$

where $\bar{y}=y-\Phi v^{(i)}$. Similarly to (20), this minimization can again be seen as an ill-posed inverse problem from measurements $\bar{y}=\Phi\left(f-v^{(i)}\right)+\varepsilon$ (in (20), $y=\gamma \Psi f$ ) with a total variation regularization. We can therefore use the extension of Chambolle's algorithm described in section 3.3.

- $u$ is fixed: one minimizes

$$
v^{(i+1)}=\underset{\tilde{v}}{\operatorname{argmin}} \mu\|\gamma \Psi \tilde{v}\|^{2}+\frac{1}{2}\|\Phi \tilde{v}-\bar{y}\|_{L^{2}}^{2}
$$

where $\bar{y}=y-\Phi u^{(i+1)}$ and where $\gamma$ are the local Fourier weights. The solution is computed by conjugate gradient descent to solve the linear system

$$
\left(2 \mu \Psi^{*} \gamma^{2} \Psi+\Phi^{*} \Phi\right) v^{(i+1)}=\Phi^{*} \bar{y},
$$

If no noise is present, then the value of $\lambda+\mu$ can be decreased during the iterations of the inpainting algorithm, in order to have a small norm for the residual term $\frac{1}{2}\|\Phi(\tilde{u}+\tilde{v})-y\|_{L^{2}}^{2}$.

\subsection{Numerical Examples}

Figure 6 presents an example of inpainting reconstruction of the image from Figure 3 degraded by randomly placed holes ( 350 squares, 15 pixels by 15 pixels, the image is of size $512 \times 512$ ). We used the same parameters as in Figure 3 . Let us notice that the texture is well reconstructed thanks to the estimation of the orientations and to the overlapping of the local FD windows. On the other hand the reconstruction of the geometric component is only accomplished by the effect of the Total Variation norm. However since our method provides a separation into two components (geometry and texture), one can imagine to apply a postprocessing on the geometric component using any method available in order to improve the final reconstruction. Figure 7 shows a second example of inpainting reconstruction for the image from Figure 4. For comparison, we also show the result of the TV diffusion process and the MCA method [17], using a curvelet dictionary for the cartoon component and a local discrete cosine transform for the texture part. For images with locally parallel patterns, our method achieves a better reconstruction of the directions of the texture inside the missing parts.

\section{Conclusion}

In this paper we presented a new adaptive texture model well-suited for locally parallel oscillating patterns. The use of this adaptive norm improves state of the art algorithms both in decomposition and inpainting for images which contain oriented textures. The adaptivity is in fact crucial for this kind of images where the texture is anisotropic, since it allows to take into account the texture geometry. 
(a) $y$

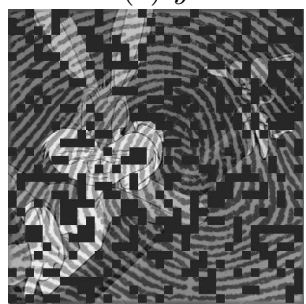

(b) $u$

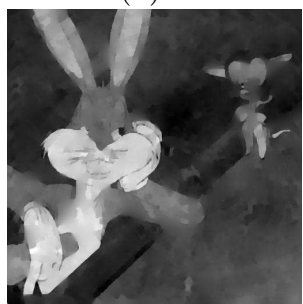

(c) $v$

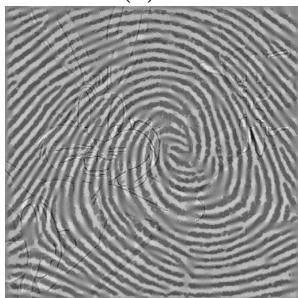

(d) $u+v$ (e) TV Inpainting

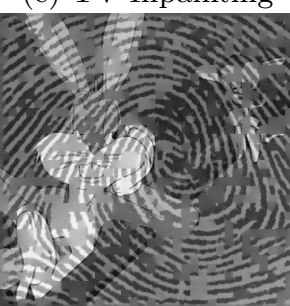

Fig. 6. First row: (a) $y$, the image to inpaint degraded by randomly chosen holes in black, the original image is $f_{0}$ in Fig. $3(\mathrm{a})$, (b) $u$ the inpainted geometric component, (c) $v$ the inpainted texture component. Second row: (d) $u+v$ the reconstruction using our method, (e) "TV Inpainting" using a simple TV diffusion.

\section{References}

1. Rudin, L.I., Osher, S., Fatemi, E.: Nonlinear total variation based noise removal algorithms. Phys. D 60(1-4) (1992) 259-268

2. Meyer, Y.: Oscillating Patterns in Image Processing and Nonlinear Evolution Equations. American Mathematical Society, Boston, MA, USA (2001)

3. Aujol, J.F., Aubert, G., Blanc-Feraud, L., Chambolle, A.: Image decomposition into a bounded variation component and an oscillating component. Journal of Mathematical Imaging and Vision 22(1) (January 2005) 71-88

4. Osher, S., Solé, A., Vese, L.: Image decomposition and restoration using total variation minimization and the $H^{-1}$ norm. Multiscale Modeling \& Simulation 1(3) (2003) 349-370

5. Nikolova, M.: A variational approach to remove outliers and impulse noise. J. Math. Imaging Vis. 20(1-2) (2004) 99-120

6. Starck, J.L., Elad, M., Donoho, D.: Redundant multiscale transforms and their application for morphological component analysis. Advances in Imaging and Electron Physics 132 (2004)

7. Masnou, S.: Disocclusion: a variational approach using level lines. IEEE Trans. Image Processing 11(2) (February 2002) 68-76

8. Bertalmio, M., Sapiro, G., Caselles, V., Ballester, C.: Image inpainting. In: Siggraph 2000. (2000) 417-424

9. Shen, J., Ha Kang, S., Chan, T.: Euler's elastica and curvature-based inpainting. SIAM Journal of Applied Mathematics 63(2) (2003) 564-592

10. Tschumperlé, D.: Fast Anisotropic Smoothing of Multi-Valued Images using Curvature-Preserving PDE's. Int. J. of Computer Vision 68(1) (2006) 65-82 
(a)
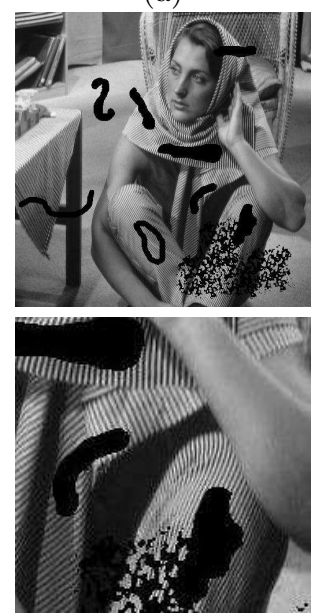

(b)

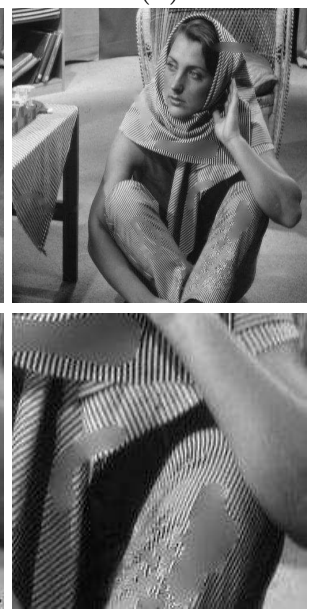

(c)

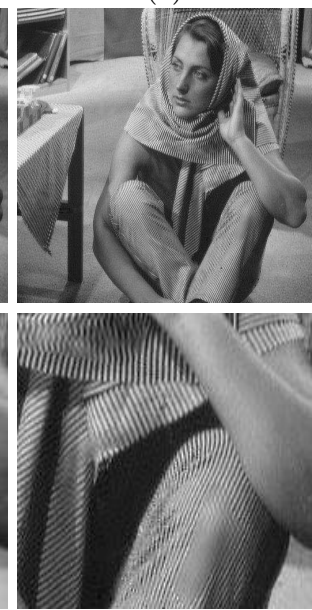

(d)

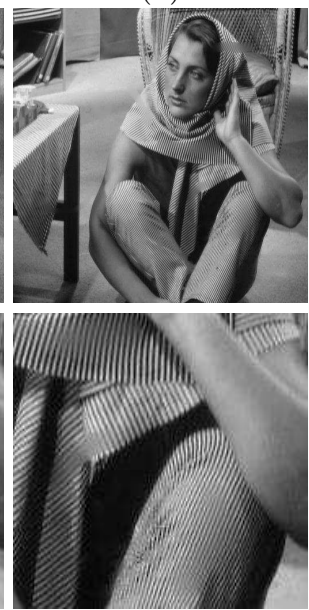

Fig. 7. Inpainting of a degraded image (the original image is $f_{0}$ in Fig. 4(a)). First row, (a) the image to inpaint, (b) reconstruction using a TV diffusion, (c) result of MCA [17] with curvelet and local discrete cosine dictionaries, (d) our reconstruction. Second row: enlargement of a part of the same images. Our method achieves a better reconstruction of the texture directions inside the missing parts.

11. Bornemann, F., März, T.: Fast image inpainting based on coherence transport. J. Math. Imaging Vis. 28(3) (2007) 259-278

12. Efros, A.A., Leung, T.K.: Texture synthesis by non-parametric sampling. In: ICCV '99. (1999) 1033

13. Wei, L.Y., Levoy, M.: Fast texture synthesis using tree-structured vector quantization. In: SIGGRAPH '00. (2000) 479-488

14. Sun, J., Yuan, L., Jia, J., Shum, H.Y.: Image completion with structure propagation. In: SIGGRAPH '05. (2005) 861-868

15. Bertalmio, M., Vese, L., Sapiro, G., Osher, S.: Simultaneous structure and texture image inpainting. IEEE Transactions on Image Processing 12 (2003) 882-889

16. Elad, M., Starck, J., Querre, P., Donoho, D.: Simultaneous cartoon and texture image inpainting using morphological component analysis (mca). Applied and Computational Harmonic Analysis 19(3) (November 2005) 340-358

17. Fadili, M., Starck, J.L.: EM algorithm for sparse representation-based image inpainting. In: IEEE Int. Conf. on Image Processing, Vol. II. (2005) 61-63

18. Aujol, J.F., Gilboa, G., Chan, T., Osher, S.: Structure-texture image decomposition-modeling, algorithms, and parameter selection. International Journal of Computer Vision 67(1) (2006) 111-136

19. Chambolle, A.: An algorithm for total variation minimization and applications. J. Math. Imaging Vis. 20 (2004) 89-97

20. Aujol, J.F.: Some first-order algorithms for total variation based image restoration. J. Math. Imaging Vis. (in press)

21. Daubechies, I., Defrise, M., Mol, C.D.: An iterative thresholding algorithm for linear inverse problems with a sparsity constraint. Commun. on Pure and Appl. Math. 57 (2004) 1413-1541 